\title{
Generative reverse-modelling approach to hygrothermal material characterization
}

\author{
Paul Klõšeiko ${ }^{1 *}$ and Peggy Freudenberg ${ }^{2}$ \\ ${ }^{1}$ Tallinn Univ. of Technology, Dept. of Civil Engin. and Arch., Ehitajate tee 5, Tallinn, Estonia \\ ${ }^{2}$ TU Dresden, Institute for Building Climatology, Zellescher Weg 17, 01069 Dresden, Germany
}

\begin{abstract}
Reliable hygrothermal modelling depends on the quality of material characterization, especially so when higher moisture contents are concerned. Previous research has shown that adding additional material tests (e.g. capillary condensation redistribution (CCR) test) to the experimental dataset brings improvements to the modelling accuracy, but also adds to the workload of characterization process. This paper discusses a generative optimization workflow to increase the speed of the characterization and quality of the result. The proposed workflow incorporates optimization tool GenOpt and hygrothermal modelling software IBK Delphin to search for best fit of the water vapour and liquid conductivity curves of interior insulation materials based on modelling the CCR, drying and wet cup tests. Finally, models using material data from the proposed workflow and from the software database are compared to measurement results from two studies on interior thermal insulation. The results suggest that the generative optimization shows promise on the grounds of reducing tedious work analysing material tests. Also, a wider experimental dataset is shown to be useful when characterizing the vapour and liquid conductivity functions in over-hygroscopic region.
\end{abstract}

\section{Introduction}

Interest in interior insulation has grown year-by-year in the Nordic countries as the need to combat high heating costs and mould risk in historic buildings increases. "Capillary active" insulation could be one solution, but the possibility of frost damage in cold climate necessitates deeper studies into the topic - also through hygrothermal modelling.

According to Künzel [1], temperature behind the insulation should be above $-5^{\circ} \mathrm{C}$ and relative humidity should be stay $\leq 95 \%_{\mathrm{RH}}$ to avoid frost damage. Hygrothermal modelling tools and material data have shown good correlation in this range and in experienced hands, the results can be considered reliable. However, these limit values restrict the thickness of interior insulation that can be applied, especially so in colder climates on walls with high thermal transmittance and where the need for thicker insulation is more pressing.

As stated in WTA Merkblatt 6-5 [2], a lot milder performance criterion can be used: the saturation degree inside the material pores should be below $30 \% \%_{\text {sat. }}$. Previous research $[3,4]$ has indicated that in high moisture content range, the modelling results can indicate much

*Corresponding author: paul.kloseiko@taltech.ee 
lower humidity than there actually is - thus making the error non-conservative. Usually, water uptake and drying test are used to determine the water vapour and liquid conductivity curves in higher moisture content range [5,6]. Assessing the share between the flows in these cases is based on assumptions as both have the same direction and are subjected to rather extreme conditions (water contact and full saturation). To gain more information on this, Binder et al $[3,7,8]$ proposed a capillary condensation redistribution (CCR) test. There, a condensation plane inside a material sample is caused by temperature difference while mass change and moisture profile are monitored.

Taking the CCR test results into account has been so far been a step-by-step iterative process [8], which adds to the already existing high work load when doing the material characterization. This paper investigates the application of a generative optimization tool GenOpt to counter that and increase the quality of the results.

\section{Methods}

The proposed workflow incorporates optimization tool GenOpt [9] and hygrothermal modelling software IBK Delphin $[10,11]$ to search for best fit of the water vapour and liquid conductivity curves of interior insulation materials based on experimental data from material tests. This work is a further development of the tool [12] used to calibrate hygrothermal simulations according to measurements.

The operating principle of the optimization tool is given in Fig. 1. Moisture dependent water vapour $\left(\log \left(K_{v}(\psi)\right)\right.$ and liquid water conductivity $\left(\log \left(K_{l}(\psi)\right)\right.$ curves are defined by having a certain number of points at fixed moisture content values while GenOpt varies their conductivities. As GenOpt handles all optimizable variables independently, the Python script checks if the liquid conductivity curve is monotonically increasing. In the case of vapour conductivity, the curve should increase until point $i$ and is expected to fall since point $j$ (both can be defined). Penalties are applied if previous conditions are not met.

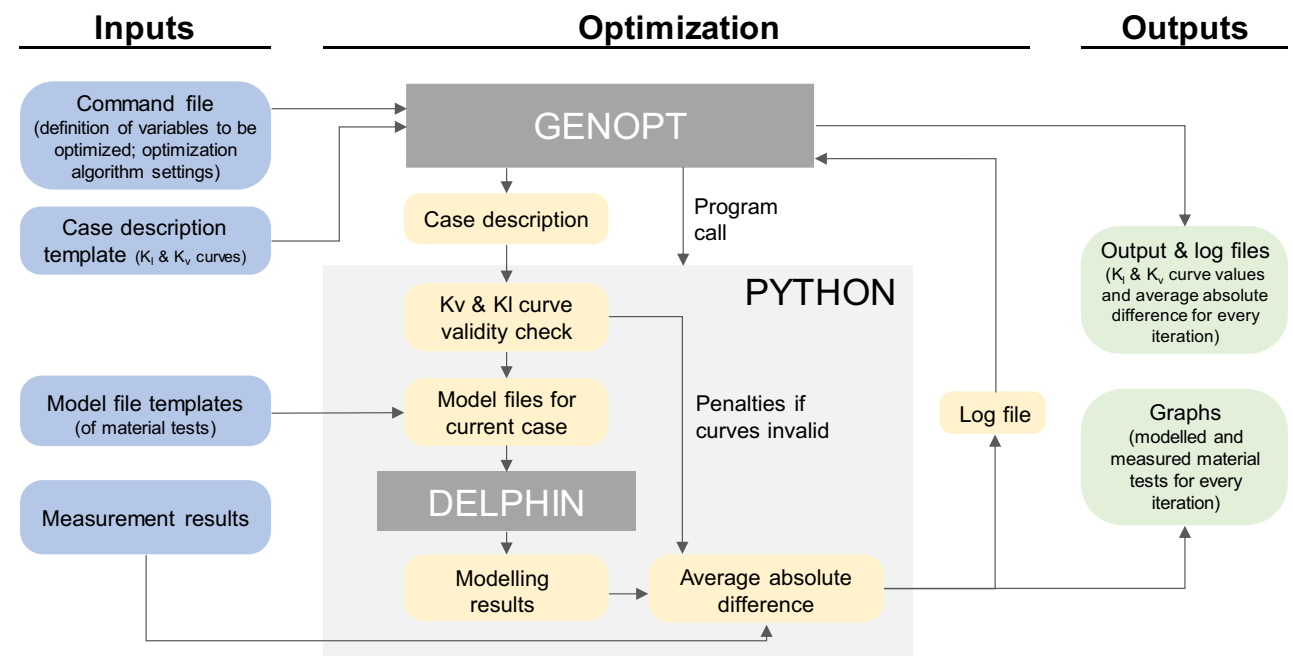

Fig. 1. Operating principle of the optimization tool based on GenOpt, Python and Delphin 5.9.

This paper presents optimization cases with autoclaved aerated concrete (AAC; mostly large macropores and fine capillaries) and calcium silicate (CaSi; mostly fine capillaries) insulation materials as an example. The tests currently incorporated in the optimization tool are: capillary condensation redistribution (CCR; moisture content profile at final time step; avg. moisture content over time), drying (avg. moisture content over time) and wet cup tests (moisture flux at wet cup boundary conditions). The CCR test data for CaSi is taken from 
[7] and for AAC from [8], the rest of the material tests are based on TU Dresden IBK lab data. First point of the $K_{v}$ curve was fixed to conductivity determined from dry cup measurements. Optimization limits were selected wide enough to keep the process unbiased. Average absolute difference between measured and modelled data is used as a cost function that GenOpt tries to minimize using Particle Swarm Optimization combined with Generalized Pattern Search method implementation of Hooke-Jeeves algorithm (GPSPSOOCCHJ)[13]. A weighting factor of 0.4 was applied to $\mathrm{CCR} \mathrm{m} / \mathrm{c}$ profile assessment (reducing its importance in overall average absolute difference) to take its larger scale into account and keep the optimization balanced. Finally, both the optimized material properties and unchanged material files from Delphin database are used to model 2 different case studies $[4,14]$ and are compared to the measurement results.

\section{Results and discussion}

\subsection{Optimization results}

In the case of $\mathrm{CaSi}, 2$ different curve resolutions were tested (high resolution (hires): $10 \mathrm{p}$ on $K_{v}$ and $15 \mathrm{p}$ on $K_{l}$ curve; low resolution (lowres): $5 \mathrm{p}$ on both $K_{v}$ and $K_{l}$ curves). For all materials, 3 initial curves were set: 1) existing Delphin curve; 2) min. limit curve; 3) max. limit curve. The $0 \ldots 0.15 \mathrm{~m}^{3} / \mathrm{m}^{3}$ part of the curves are shown in Fig. 2. Compared to Delphin versions, the optimized curves tend to have lower liquid conductivity and higher vapour conductivity. Table 1 gives an overview of final assessment value and time consumption of the optimization cases. Fig. 3 shows the results of material tests that the optimization was based upon. The drying test is modelled well with all material properties. The biggest differences are in CCR test results - optimized materials manage to reproduce the high moisture content at cold side of the sample. Optimized CaSi has problems reaching the "dynamic equilibrium" when comparing the average $\mathrm{m} / \mathrm{c}$ of CCR test.
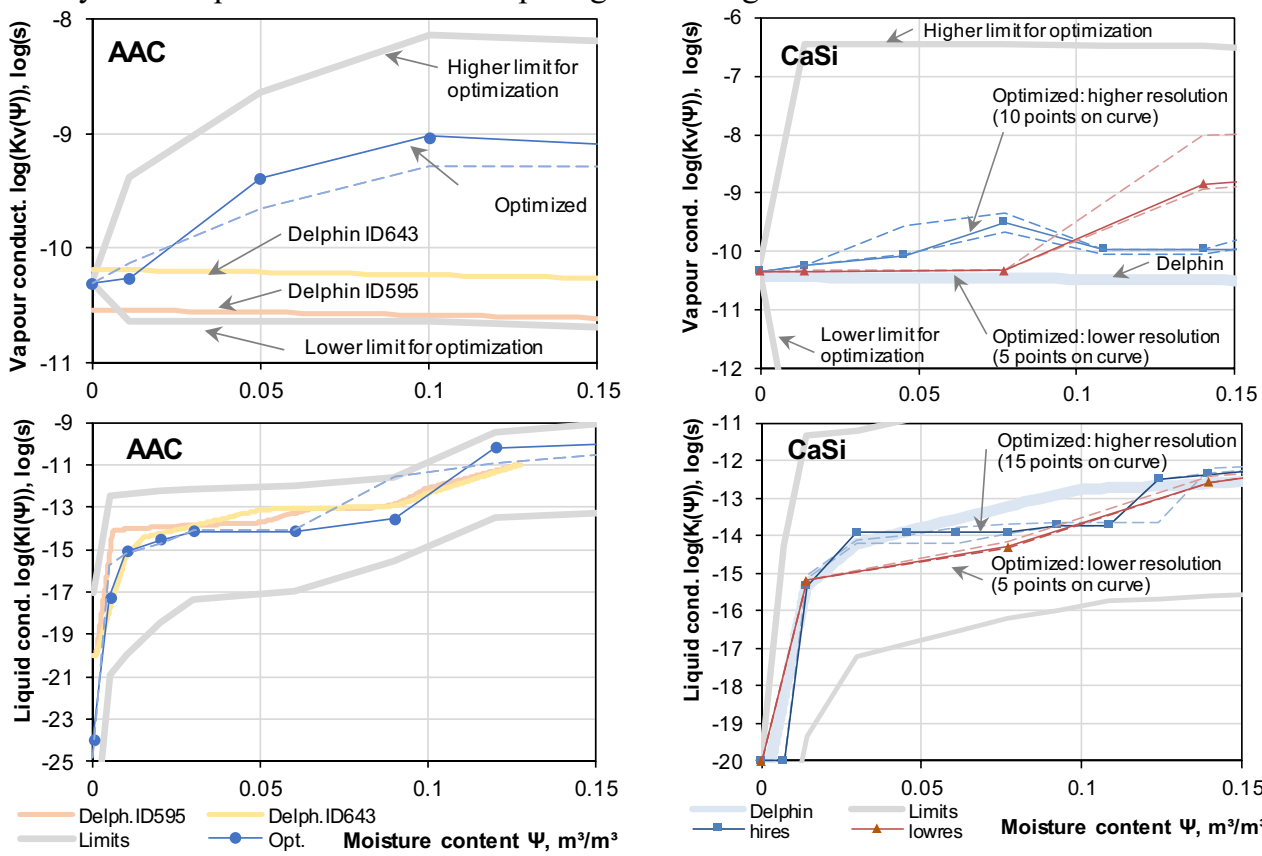

Fig. 2. Comparison of optimized and Delphin versions of liquid (top row) and vapour conductivity curves (bottom row). Dashed lines represent cases where initial values of the variables were different. 
Table 1. Optimization cases and their properties. Cases chosen for further use have been underlined.

Case

CaSi highres

CaSi highres.min

CaSi highres.max

CaSi lowres

CaSi lowres.min

CaSi lowres.max

$\underline{\mathrm{AAC}}$

AAC.min

AAC.max

Initial curves

* - curve not conforming to the set rules in 1 point
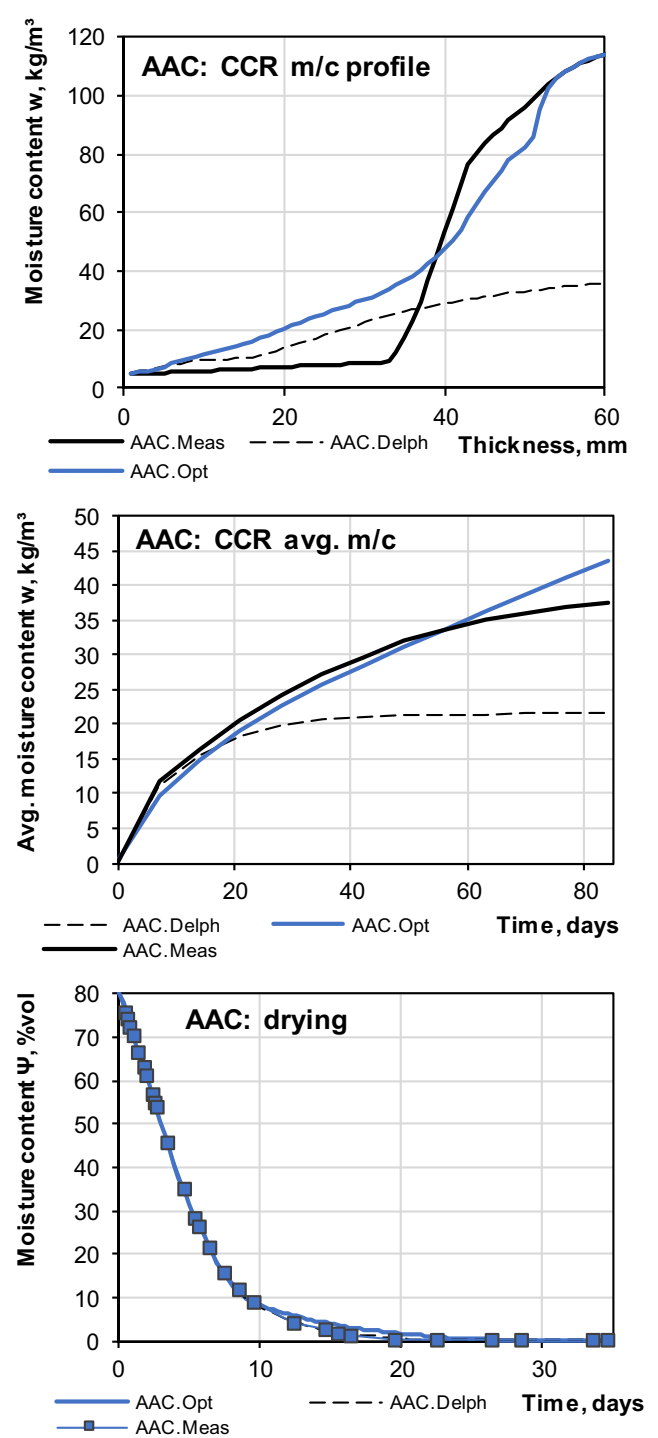

Avg. abs. diff. Iterations Run time, $h$

min. limit

$\underline{2.72}$

$\underline{7000}$

6541

$\underline{18}$

$2.72 *$

6315

$2.80^{*}$

$\underline{2.74}$

2.81

$\underline{2260}$

2988

2.74

2991

$\underline{1.72}$

2.21

$\underline{2685}$

1948

2.21

1951
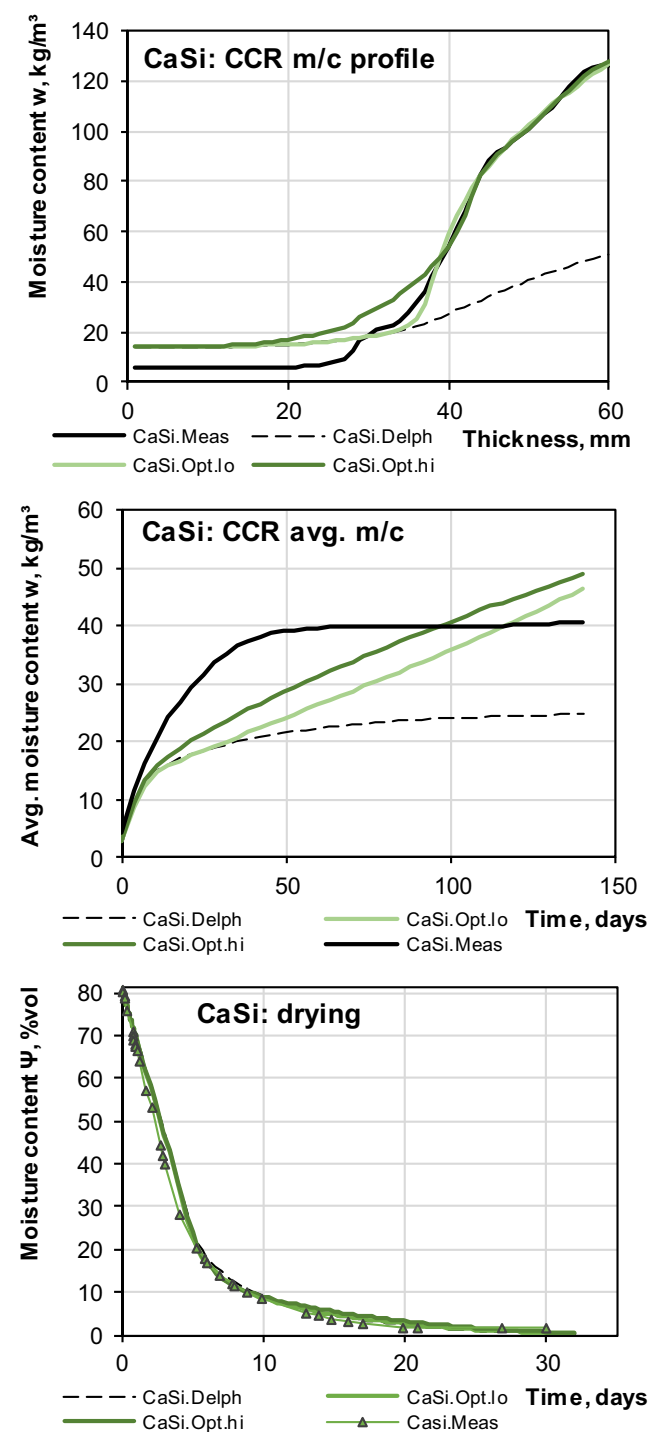

Fig. 3. Comparison of measured and modelled material test results for AAC (left) and CaSi (right). 


\subsection{Comparison to case studies}

Two case studies were used to compare how the optimized material properties behaved against their unchanged versions and the measurement results. The first one [4] took place in a climate chamber and is characterized by high moisture contents. Also, during the second half of the study, $\sim 50$ freeze-thaw cycles take place. Fig. 4 gives the modelled and measured moisture content of $\sim 7 \mathrm{~mm}$ layer on the cold side of insulation. Moisture contents of AAC and CaSi are both underestimated by the Delphin database versions of the material data, while the optimized version of AAC is remarkably closer to measured results.
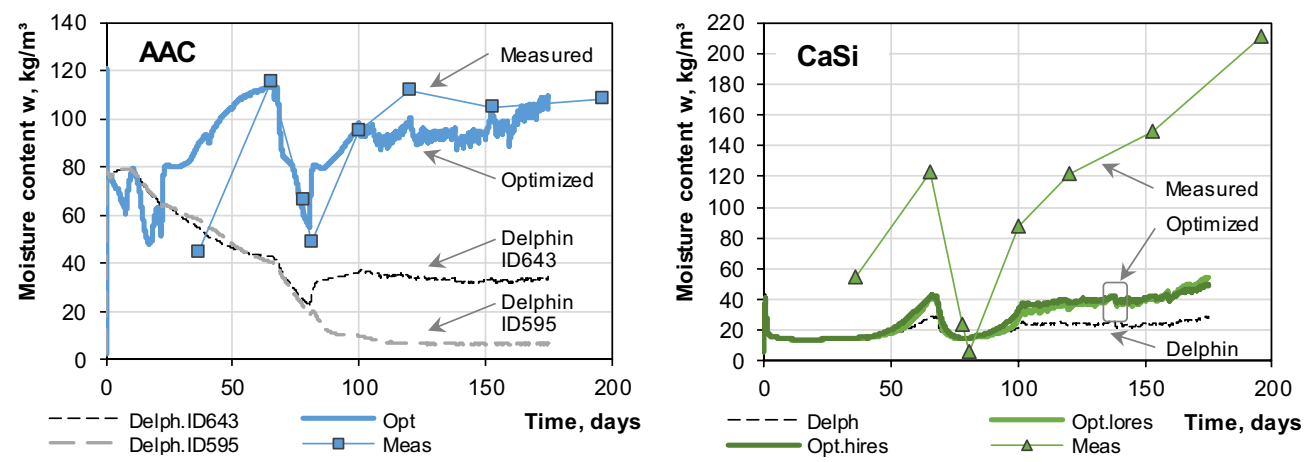

Fig. 4. Case study 1 (high moisture content; climate chamber): comparison of measurement results to modelling with optimized and unchanged material properties for AAC (left) and $\mathrm{CaSi}$ (right).

The second case study [14] was based on an exterior brick wall of a historic school building with added interior insulation and a humidification system. The moisture load mimicked an unventilated apartment. Moisture was measured using relative humidity sensors. Fig. 5 shows that the the optimized and database versions of material properties result in similar $\mathrm{RH}$ values for both $\mathrm{AAC}$ and $\mathrm{CaSi}$.
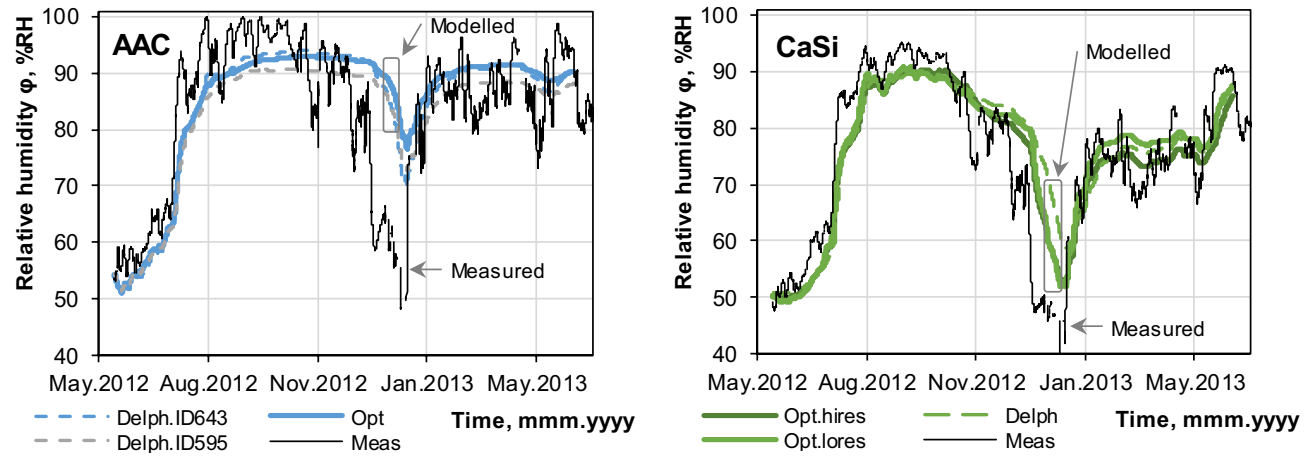

Fig. 5. Case study 2 (medium moisture content; real building): comparison of measurement results to modelling with optimized and unchanged material properties for AAC (left) and $\mathrm{CaSi}$ (right).

\section{Conclusions}

As a study on developing a material characterization tool, the results can be considered good. Although the variation limit boundaries were kept large and different initial values and curve resolutions were used for optimization, the resulting optimized curves were in a relatively similar range and in most part matched the curves in Delphin database. However, optimized liquid conductivity tended to be lower and vapour conductivity higher in the range of $0.05 \ldots 0.1 \mathrm{~m}^{3} / \mathrm{m}^{3}$ moisture content. This could be explained by the liquid island 
effect. Still, as the CCR test data was taken from literature and the exact tested products and boundary conditions were not disclosed, it is quite possible that the CCR and rest of material data were measured from different products. This brings along mismatches in moisture retention curves and the optimization algorithm trying to compensate for it through conductivity curves.

Comparison to case study results show that for both AAC and $\mathrm{CaSi}$, the optimized materials show better correlation to measured data in high moisture content range (especially so for $\mathrm{AAC}$ ), hinting at the value of CCR test. In hygroscopic range, the optimized properties perform similarly to their default counterparts - as Delphin has shown to be quite reliable in that range, it could be considered a good result for the algorithm. These results underline that there are still many unknowns when dealing with overhygroscopic moisture contents - caution is advised when drawing far-reaching conclusions from modelling results in that region.

Future work could include developing a wider experimental base for optimization and defining stricter rules/boundaries for the conductivity curves. Currently, the results show quite high spread at moisture contents above CCR test - the share of liquid and vapour transfer is hard to determine. Also, the CCR test could be enhanced with relative humidity sensors in the samples to gain more reference data from the hygroscopic region.

The research is supported by personal research funding grant PRG483, Moisture safety of interior insulation, constructional moisture and thermally efficient building envelope and by the European Union through European Regional Development Fund.

\section{References}

1. H. M. Künzel, in 1. Internationaler Innendämmkongress 2011, 2011, 9-16.

2. H. Künzel et al., 2012.

3. A. Binder, D. Zirkelbach, and H. M. Künzel, in Thermal Performance of the Exterior Envelopes of Whole Buildings XI, 2010.

4. P. Klõšeiko, K. Varda, and T. Kalamees, Energy Procedia 132, 525-530 (2017).

5. G. a. Scheffler and R. Plagge, Int. J. Heat Mass Transf. 53, 286-296 (2010).

6. M. Krus, Moisture transport and storage coefficients of porous mineral building materials. Theoretical principles and new test methods. Stuttgart: Fraunhofer IRB Verlag, 1996.

7. A. Binder, H. M. Künzel, and D. Zirkelbach, in Proceedings of 2nd Central European Symposium on Building Physics, 2013.

8. A. Binder, D. Zirkelbach, and H. M. Künzel, in NSB 2014, 10th Nordic Symposium on Building Physics: 15-19 June 2014, Lund, Sweden, 2014, 1061-1068.

9. M. Wetter, in Seventh International IBPSA Conference, 2001.

10. A. Nicolai, G. A. Scheffler, J. Grunewald, and R. Plagge, in Simulation of Time Dependent Degradation of Porous Materials, 2009.

11. J. Grunewald, TU Dresden, 1997.

12. P. Freudenberg, U. Ruisinger, and E. Stöcker, Energy Procedia 132, 405-410 (2017).

13. M. Wetter, GenOpt ${ }^{\circledR}$ User Manual v3.1.1. Berkeley: LBNL, 2016.

14. P. Klõšeiko, E. Arumägi, and T. Kalamees, J. Build. Phys. 38, 444-464 (2015). 